\title{
Face Detection Using Skin Tone Segmentation
}

\author{
Sayantan Thakur ${ }^{1}$, Sayantanu Paul ${ }^{1}$, Ankur Mondal $^{2}$ \\ ${ }^{1}$ Department of Information Technology \\ ${ }^{2}$ Department of Computer Science and Engineering \\ Guru Nanak Institute of Technology \\ Sodepur, Kolkata- 700 114, India \\ e-mail: sayantan.thakur@gmail.com, \\ sayantanu_paul@yahoo.co.in, mondal.ankur@gmail.com
}

\author{
Swagatam Das \\ Electronics and Communication Science Unit \\ Indian Statistical Institute \\ Kolkata 700108, India \\ e-mail: swagatam.das@isical.ac.in
}

\author{
Ajith Abraham \\ Machine Intelligence Research Labs (MIR Labs) \\ Auburn, Washington 98071-2259, USA \\ e-mail: ajith.abraham@ieee.org
}

\begin{abstract}
Human face detection has become a major field of interest in current research because there is no deterministic algorithm to find face(s) in a given image. Further the algorithms that exist are very much specific to the kind of images they would take as input and detect faces. The problem is to detect faces in the given, colored group photograph. In this paper, an improved segmentation algorithm for face detection in color images with multiple faces and skin tone regions is proposed. Algorithm ingeniously uses a novel skin color model, RGB-HS-CbCr for the detection of human faces. Skin regions are extracted using a set of bounding rules based on the skin color distribution obtained from a training set. The segmented face regions are further classified using a parallel combination of simple morphological operations. Experimental results on a large photo data set have demonstrated that the proposed model is able to achieve good detection success rates for near-frontal faces of varying orientations, skin color and background environment.
\end{abstract}

Keywords---Face detection; skin color; color models; skin classification; bounding box; eccentricity

\section{INTRODUCTION}

Detection of the human face is an essential step in computer vision and many biometric applications [1] such as automatic face recognition, video surveillance [2], human-computer communication and large-scale face image retrieval systems. The first and foremost important step in any of these systems is the accurate detection of the presence and the position of the human faces in an image [3] or video. The main challenges encountered in face detection is to cope with a wide variety of variations in the human face such as posture and scale, face orientation, facial expression, ethnicity and skin color $[3,4,5]$. External factors such as occlusion, complex backgrounds $[6,7,8]$ inconsistent illumination conditions and quality of the image may also contribute significantly to the overall problem. Face detection in color images [1,9] has also gained much attention and notice in recent years. Color is known to be a useful cue to extract skin regions $[2,10,11]$ and it is only available in color images. This allows easy face localization of potential facial regions without any consideration of its texture and geometrical properties $[8,12]$. Most techniques up to date are pixel-based skin detection methods [13], which classify each pixel as skin or "non-skin" individually and independently from its neighbors. Some of the early methods used various statistical color models such as a single Gaussian model [14], Gaussian mixture density model [15] and histogrambased model [16].

Various colors spaces provides us various discriminality between skin pixels and non-skin pixels over various illumination conditions. Skin color models [17] that operate only on chrominance subspaces such as the $\mathrm{Cb}-\mathrm{Cr}[18,19,20]$ and $\mathrm{H}-\mathrm{S}$ [21] have been found to be effective in characterizing various human skin colors. Peer et al.[22]constructed a set of rules to describe skin cluster in RGB space while Garcia and Tziritas [23] used a set of bounding rules to classify skin regions on both $\mathrm{YCbCr}[28]$ and HSV spaces.

In this paper, we present a novel skin color model RGB-HS-CbCr for human face detection]. This model utilizes the additional hue and chrominance information of the image on top of standard RGB properties to improve the discriminality between skin pixels and non-skin pixels. In our approach, skin regions are classified using the RGB boundary rules introduced by Peer et al. [22] and also additional new rules for the $\mathrm{HS}$ and $\mathrm{CbCr}$ subspaces. These rules are constructed based on the skin color distribution obtained from the training images. The classification of the extracted regions is further refined using a parallel combination of morphological operations. 
The rest of the paper is organized as follows: Section 2 briefly describes the various steps of our face detection system. The construction of the RGB-HS-CbCr skin color model is described in Section 3. Section 4 presents the use of morphological operations in our algorithm [30,33]. Section 5 represents the pseudo code of the system Experimental results and discussions are provided in Section 6. Finally, Section 7 concludes the paper.

\section{SYSTEM OVERVIEW}

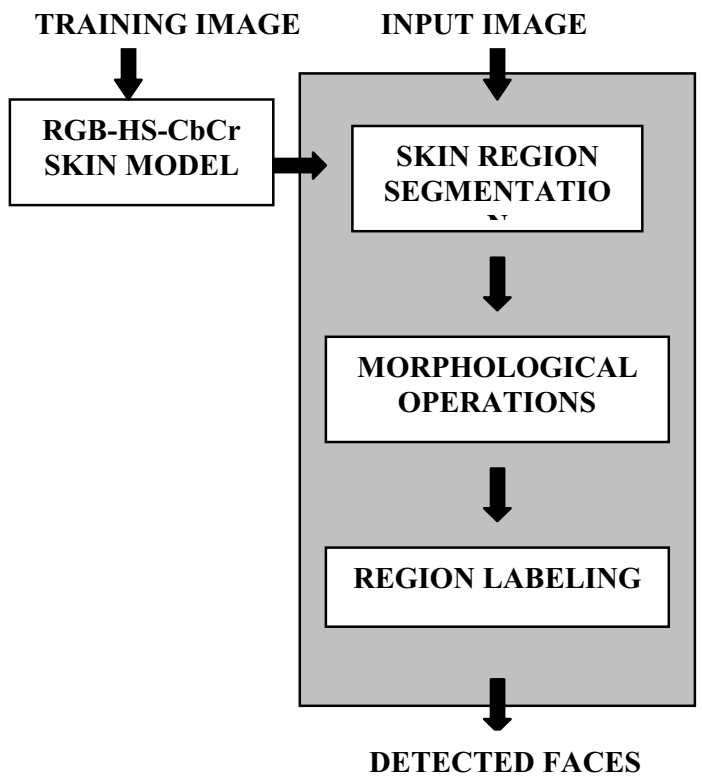

Figure 1. System overview of Face Detection System

Fig:1 shows the system overview of the proposed face detection system, which consists of a training stage and detection stage. In our color-based approach to face detection, prior formulation of the proposed RGB-HS$\mathrm{CbCr}$ skin model is done using a set of skin-cropped training images. Three commonly known color spaces$\mathrm{RGB}, \mathrm{HSV}$ and $\mathrm{YCbCr}$ are used to construct the proposed hybrid model. Bounding planes or rules for each skin color subspace are constructed from their respective skin color distributions.

In the first step of the detection stage, these bounding rules are used to segment the skin regions of input test images.After that,a combination of morphological operations are applied to the extracted skin regions to eliminate possible non-face skin regions. Finally, the last step labels all the face regions in the image and returns them as detected faces. In our system, there is no preprocessing step as it is intended that the input images are thoroughly tested under different image conditions such as illumination variation and quality of image. Since we emphasize on the use of a novel skin color model in this work, our system is restricted to color images only.

Fig 1 : System overview of Face Detection System

\section{RGB-HS-CBCR MODEL}

\section{A. Preparations of training images}

In order to build the skin color model [17], a set of training images were used to analyze the properties and distribution of skin color in various color subspaces.

Our training image set composed of twenty color images obtained from the Internet, covering a wide range of variations (different ethnicity and skin color). These images contained skin color regions that were either exposed to normal uniform illumination, daylight illumination (outdoors) or flashlight illumination (under dark conditions) Fig. 2 shows two samples of the skin detected training.
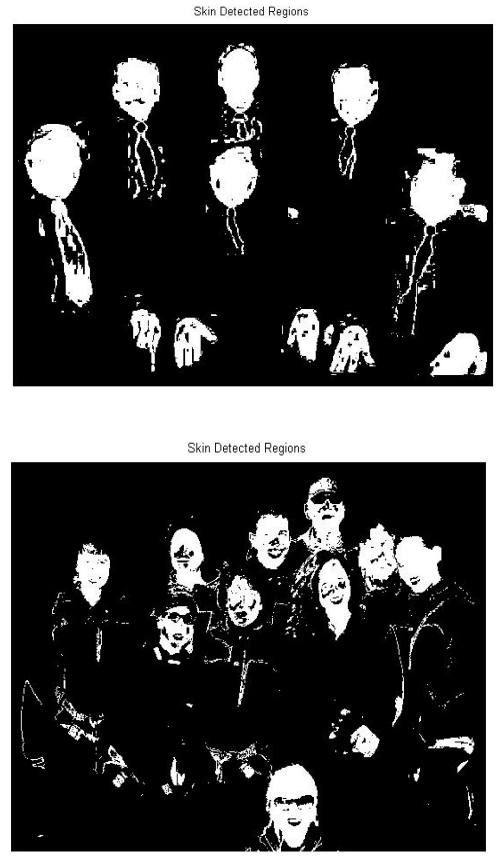

Figure 2. Skin Detected training images

\section{B. Skin color bounding rules}

The training images were analyzed in the RGB, HSV and $\mathrm{YCbCr}$ spaces. In RGB space, the skin color region is not well-distinguished in all 3 channels. A simple observation of its histogram will show that it is uniformly spread across spectrum of values.

In HSV space, the $\mathrm{H}$ (Hue) channel shows significant discrimination of skin color regions.

In RGB space, we use the skin color rules introduced by Peer et al. [22].The skin color at uniform daylight illumination rule is defined as

$(\mathrm{R}>50) \& \&(\mathrm{G}>40) \& \&(\mathrm{~B}>20) \& \&[\max \{\max (\mathrm{R}, \mathrm{G}), \mathrm{B}\}-$ $\min \{\min (R, G), B\}]>10) \& \&(R-G>=10) \& \&(R>G)$ $\& \&(\mathrm{R}>\mathrm{B})$ 
while the skin color under flashlight or daylight lateral illumination rule is given by

$(R>220) \& \&(G>210) \& \&(B>170) \& \&(|R-G| \leq 15) \& \&$ $(\mathrm{R}>\mathrm{B}) \& \&(\mathrm{G}>\mathrm{B})$

To consider both conditions when needed, we used a logical OR to combine both (1) and (2). The RGB bounding rule is denoted as Rule $\mathrm{A}$.

Rule A: Equation(1) $\cup$ Equation(2)

Based on the observation that the $\mathrm{Cb}-\mathrm{Cr}$ subspace is a strong discriminate of skin color, we formulated 2 bounding planes. The two bounding rules that enclosed the $\mathrm{Cb}-\mathrm{Cr}$ skin color region are formulated as below:

$\mathrm{C}_{\mathrm{B}} \geq 60 \& \& \mathrm{C}_{\mathrm{R}} \leq 130$

$\mathrm{C}_{\mathrm{B}} \geq 130 \& \& \mathrm{C}_{\mathrm{R}} \leq 165$

Rule (4) and (5) are combined using a logical $\mathrm{AND}(\& \&)$ to obtain the $\mathrm{C}_{\mathrm{B}} \mathrm{C}_{\mathrm{R}}$ bounding rule, denoted by Rule B.

Rule B: Equation(4) กEquation(5)

In the HSV space, the hue and saturation values exhibit the most noticeable separation between skin and non-skin regions. We estimated two cutoff levels as our HS subspace skin boundaries,

$\mathrm{H} \geq 0 \quad \& \& \quad \mathrm{H} \leq 50$

$\mathrm{S} \geq 0.1 \& \& \mathrm{~S} \leq 0.9$

where both rules are combined by a logical AND to obtain the $\mathrm{H}$ bounding rule, denoted as Rule $\mathrm{C}$.

Rule C: Equation(7) $\cap$ Equation(8)

Thereafter, each pixel that fulfills Rule A, Rule B and Rule $\mathrm{C}$ is classified as a skin color pixel,

Rule $A \cap$ Rule $B \cap$ Rule $C$

\section{Skin color segmentation}

The proposed novel combination of all 3 bounding rules from the RGB, HS and $\mathrm{CbCr}$ subspaces (Equation $10)$ is named the "RGB-HS-CbCr" skin color model. Although skin color segmentation [25,27,29,31,32] is normally considered to be a low-level or "first-hand" cue extraction, it is crucial that the skin regions are segmented precisely and accurately. Our segmentation technique, which uses all 3 color spaces was designed to boost the face detection accuracy, as will be discussed in the experimental results. Fig. 7 shows the skin segmentation result of two sample test images using the RGB-HS- $\mathrm{CbCr}$ model.

The resulting segmented skin color regions have three common issues:

a) Regions are fragmented and often contain holesand gaps.

b) Occluded faces or multiple faces of closeproximity may result in erroneous labeling (e.g. agroup of faces segmented as one).

c) Extracted skin color regions may not necessarily be face regions. There are possibilities that certain skin regions may belong to exposed limbs (arms and legs) and also foreground and background objects that have a high degree of similarity to skin color.

\section{MORPHOLOGICAL OPERATIONS}

The next step of the face detection system involves the use of morphological operations to refine the skin regions extracted from the segmentation step.

The first step that we include is a simple hole filling operation to fill any holes [26] or gaps in the individual blobs. For opening the narrowly connected blobs we subtract the image obtained from bwperim function [24] from the main binary image for a specified number of times, thus finally separating the narrowly connected regions. As the subtraction leads to loss in size of the individual blobs our final morphological operation includes a dilate function [24] to maintain a particular size of the skin detected individual blobs.

Additional measures are also introduced to determine the likelihood of a skin region being a face region. Two region properties - box ratio and eccentricity are used to examine and classify the shape of each skin region.

The box ratio [24] property is simply defined as the width to height ratio of the region bounding box. By trial and error on the training set we have found that the good range of values lie between 1.1 and 0.4 . Ratio values above 1.1 would not suggest a face since human faces are oriented vertically with a longer height than width. Meanwhile, ratio values below 0.4 are found to misclassify arms, legs or other elongated objects as faces.

The eccentricity property measures the ratio of the minor axis to major axis of a bounding ellipse. Eccentricity values of between 0.25 and 0.97 are estimated to be of good range for classifying face regions. Though this property works in a similar way as box ratio, it is more sensitive to the region shape and is able to consider various face rotations and poses.

Fig3, Fig4 and Fig5 respectively show the images after eliminating connected regions, after applying bounding box \& eccentricity and final detected face. 


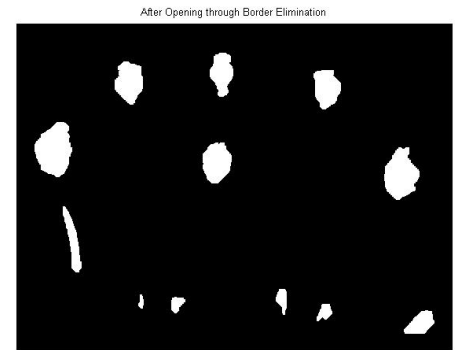

Figure 3. Connected Region eliminated training image

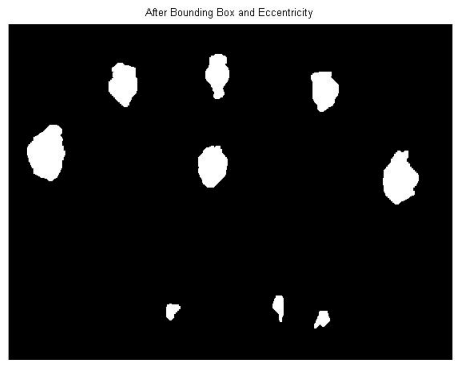

Figure 4. Traing image after applying Bounding Box and Eccentricity

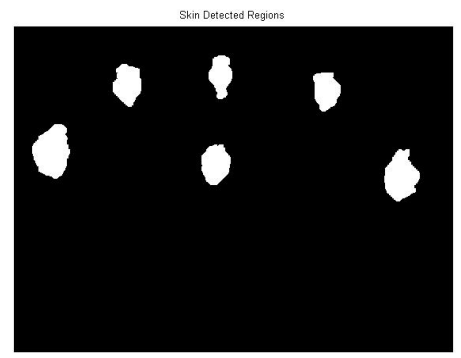

Figure 5. Face detected training image

\section{PSEUDO CODE}

Step 1: Read the image file, picture.

Step 2: Initialize value R, G, B as the red, green and blue values respectively of picture.

Step 3: Initialize value $\mathrm{H}, \mathrm{S}$ as the hue and saturation values respectively of picture.

Step 4: Initialize value $C_{b}$ and $C_{r}$ as the blue-difference and red-difference values respectively of picture.

IF ( $(R>50 \& G>40 \& B>20 \&(\max (R, G, B)-\min (R, G$, B) $)>10 \&$ abs $(R-G)>=10 \& R>G \quad \& \quad R>B)$ OR $(R>220 \&$ $\mathrm{G}>210 \quad \& \quad \mathrm{~B}>170 \quad \& \operatorname{abs}(\mathrm{R}-\mathrm{G})<=15 \quad \& \quad \mathrm{R}>\mathrm{B} \quad \& \quad \mathrm{G}>\mathrm{B})$ THEN

$$
\begin{gathered}
\text { IF }\left(\mathrm{C}_{\mathrm{b}}>=60 \& \mathrm{C}_{\mathrm{b}}<=130 \& \mathrm{C}_{\mathrm{r}}>=130 \& \mathrm{C}_{\mathrm{r}}<=165\right) \\
\text { THEN } \\
\text { IF }(\mathrm{H}>=0 \& \mathrm{H}<=50 \& \mathrm{~S}>=0.1 \& \quad \mathrm{~S}<=0.9)
\end{gathered}
$$

THEN

Mark as skin region

ELSE

REJECT that pixel. ENDIF

\section{ENDIF}

Step 5: Set blob_pixels as the number of pixels in each connected region.

\section{IF blob_pixels $<1500$ THEN \\ REJECT that connected region. \\ ENDIF}

Step 6: Perform hole filling operation and "bwperim" to open up narrowly connected regions.

FOR each connected region

SET breadth as the breadth of the bounding box of the region.

SET length as the length of the bounding box of the region.

SET ratio=breadth/length of the bounding box of the region.

SET eccen as the eccentricity of the bounding box of the region.

IF $($ ratio $<0.4 \|$ ratio $>1.1) \|($ eccen $<0.25 \|$ eccen $>0.97)$ THEN

REJECT that connected region

\section{ENDIF}

END FOR

Step 7: FOR each region remaining

Draw a red rectangular box on the region for face detection

END FOR

Step 8: DISPLAY the output image, with the face detection.

\section{EXPERIMENTAL RESULTS AND DISCUSSIONS}

The proposed RGB-HS-CbCr skin color model for skin region segmentation was evaluated on a face detection system using a test data set of 16 images, containing a total of 100 unique faces. In order to build this test data set, the images were randomly selected from the Internet, each comprising of two or more near-frontal faces and of a large variety of descent (Asians, Caucasians, Middle-Eastern, Hispanic and African). The test images also consist of various indoor and outdoor scenes and of different lighting condition-daylight, fluorescent light, flash light (from cameras) or a combination of them. The face detection system was implemented using MATLAB on a $2.4 \mathrm{MHz}$ Pentium IV machine running on $1024 \mathrm{MB}$ RAM. 
To evaluate our experiments, we defined two performance metrics to gauge the success of our schemes. False Detection Count(FDC) is defined as the number of false detections over the total number of detections.

$$
\mathrm{FDC}=\frac{\text { no. } \text { of false detections }}{-\mathrm{total} \text { number of detections }} \times 100 \%
$$

Detection Success Count (DSC) is defined as the number of correctly detected faces over the actual number of faces in the image.

$$
\mathrm{DSC}=\frac{\text { no. of correctly detected faces }}{\text { total number of faces }}
$$

where the number of correctly detected faces is equivalent to the number of faces minus the number of false dismissals.

To evaluate the effectiveness of the RGB-HS-CbCr skin color model, the face detection system was tested with various combination of color models, each represented by its own set of bounding rules. The combination of all 3 subspaces resulted in the best DSC and lowest FDC values. Section VI(A) presents some sample results of the proposed face detection system. TableI shows data obtained from our training images.

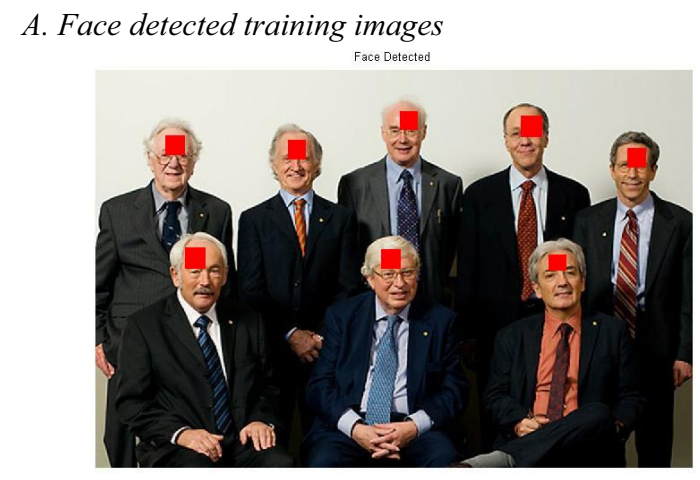

Figure 6.

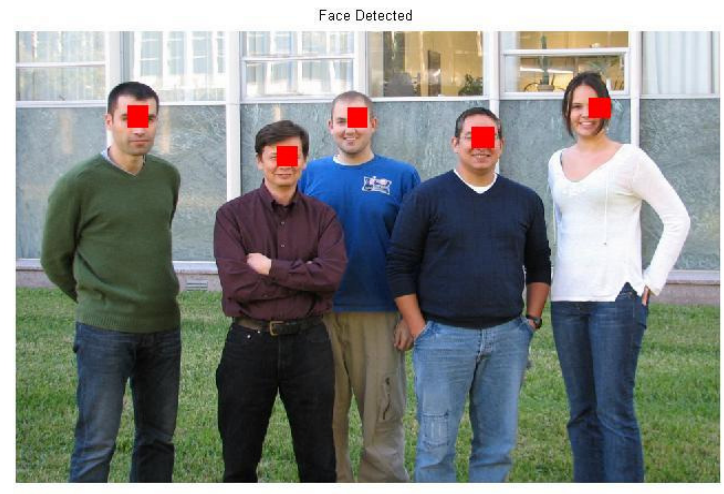

Figure 7.

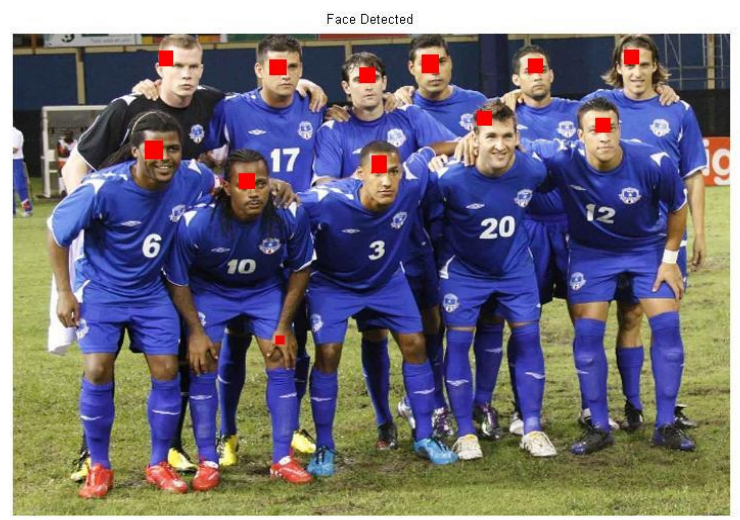

Figure 8.

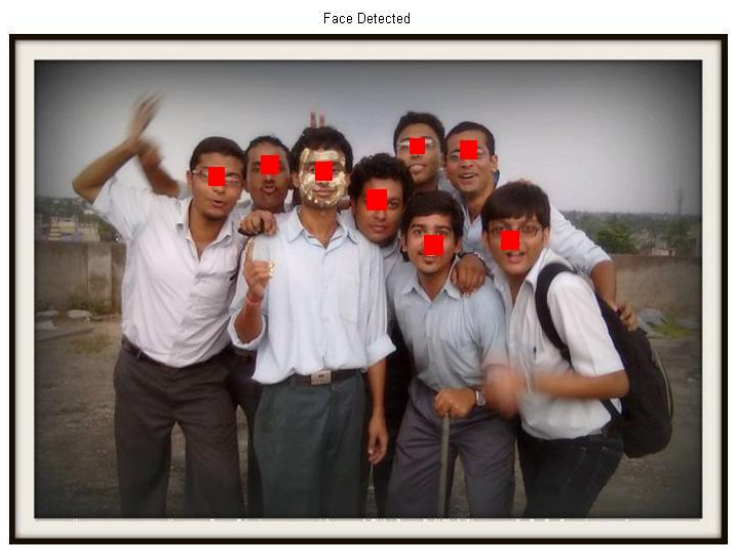

Figure 9. 
Face Detected

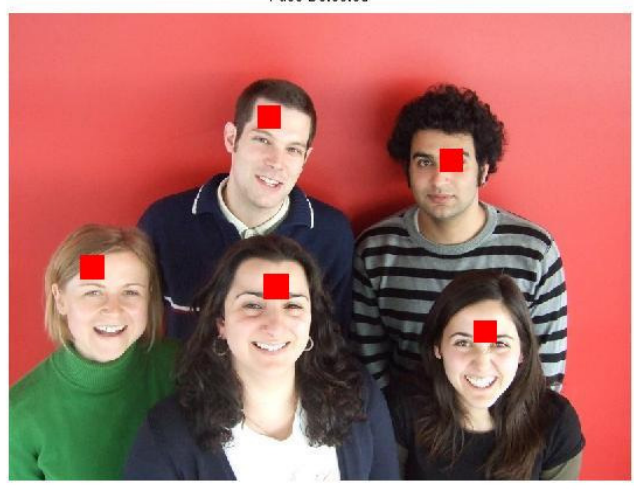

Figure 10.

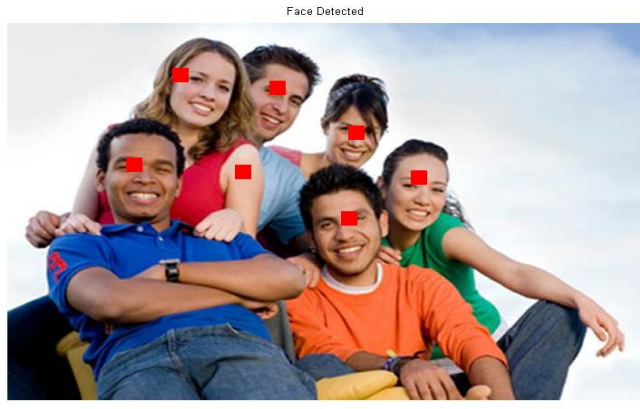

Figure 11

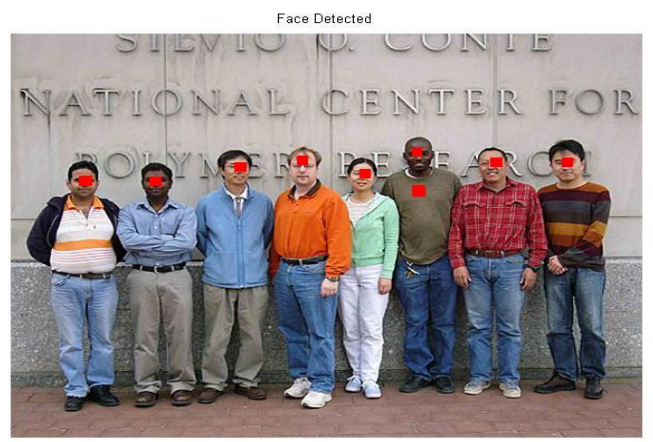

Figure 12.

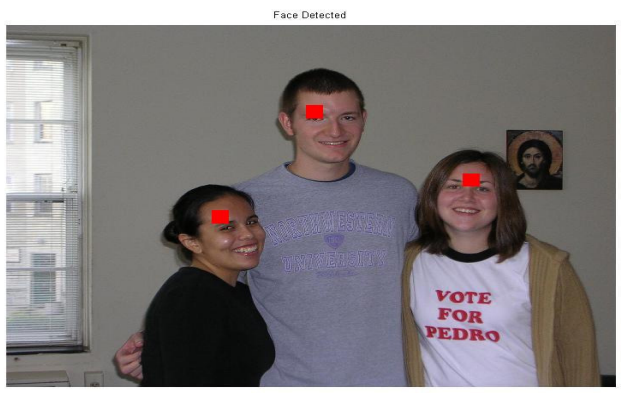

Figure 13.
Face Detected

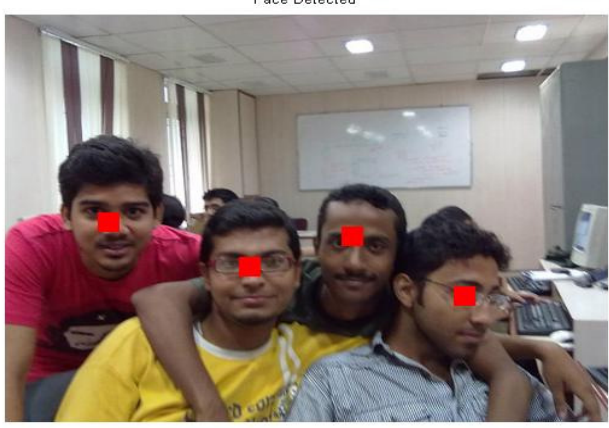

Figure 14.

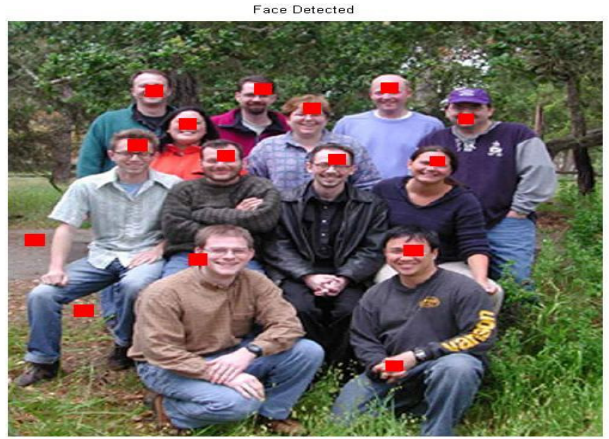

Figure 15.

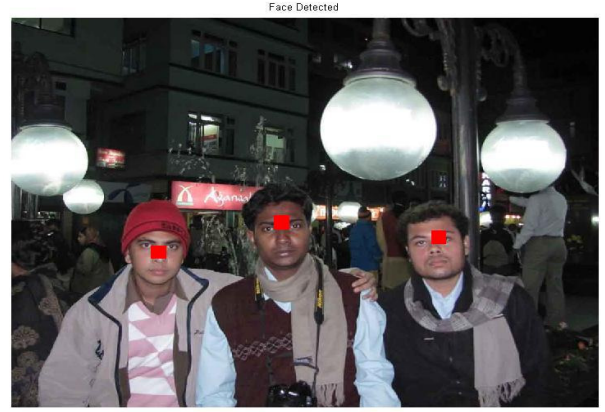

Figure 16.

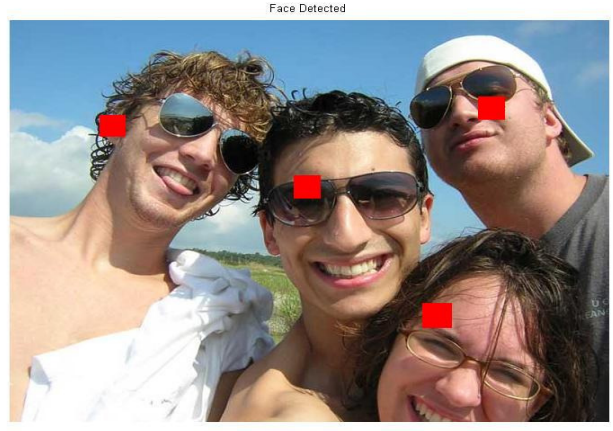

Figure 17. 


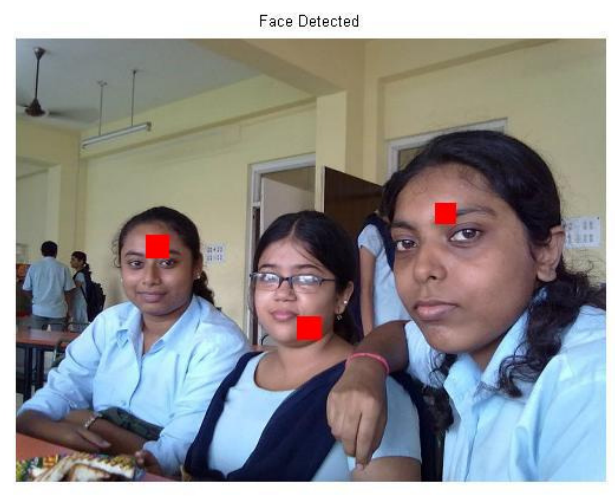

Figure 18.

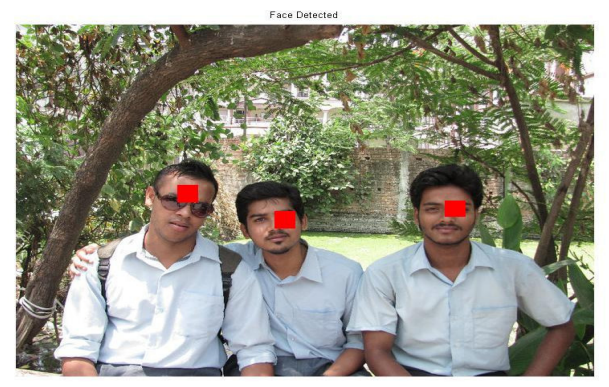

Figure 19.

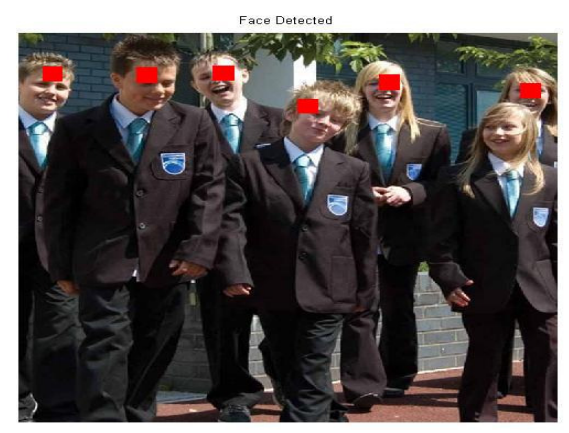

Figure 20.

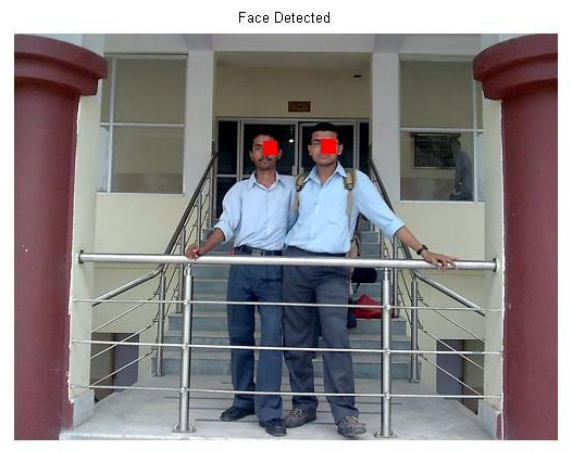

Figure 21.

TABLE I. DATA OBTAINED FROM TRAINING IMAGES

FIGURE FDC $(\%)$ $\operatorname{DSC}(\%)$
6

7

8

9

10

11

12

13

14

15

16

17

18

19

20

21

$\begin{array}{lll} & \text { FDC(\%) } & \text { DSC(\%) } \\ 0 & 100 \\ 0 & 100 \\ 8.33 & 100 \\ 0 & 100 \\ 0 & 100 \\ 14.2 & 100 \\ 11.11 & 100 \\ 0 & 100 \\ 0 & 100 \\ 20 & 100 \\ 0 & 100 \\ 0 & 100 \\ 0 & 100 \\ 0 & 100 \\ 0 & 85.7 \\ 0 & 100\end{array}$

\section{CONCLUSION}

Even though there are many modern and accurate softwares today yet in this paper, we have presented a novel skin color model, RGB-HS-CbCr to detect human faces for students with minimal knowledge in image processing. The Skin region segmentation was performed using combination of RGB, HS and $\mathrm{CbCr}$ subspaces, which demonstrated evident discrimination between skin and non-skin regions. The experimental results showed that our new approach in modeling skin color was able to achieve a good detection success rate.

This project would serve as a stepping stone for future improvements and modifications. As it is simple to understand hence it will readily understood by students who then will be encouraged to make modifications and create a more sophisticated project.

\section{ACKNOWLEDGMENT}

At very first we would like to express our profound gratitude to Dr. Arup $\mathrm{Kr}$. Bhaumik, principal of Gurunanak Institute of Technology for huge encouragement and constant support for research work. Next we must be thankful to the Electronics and Communication Science Unit of the Indian Statistical Institute for wonderful work environment. And at last but not the least we are very much grateful to all members of the MIR labs for continuous knowledge upgrading and sharing with everyone from student level to professor level.

\section{REFERENCES}

[1] R.-L. Hsu, M. Abdel-Mottaleb, and A. K. Jain, "Face detection in color images," IEEE Trans. PAMI, vol. 24, no. 5, pp. 696-707, 2002.

[2] U.A. Khan, M.I. Cheema and N.M. Sheikh, "Adaptive video encoding based on skin tone region detection," in: Proceedings of 
IEEE Students Conference, Pakistan, 16-17 August 2002, vol (1), pp. 129-34.

[3] S. L. Phung, D. Chai, and A. Bouzerdoum, "A universal and robust human skin color model using neural networks," Proc. IJCNN'01, July 2001, pp. $2844-2849$.

[4] S. L. Phung, A. Bouzerdoum, and D. Chai, "Adaptive skin segmentation in color images," Proc. ICASSP'03, 2003.

[5] Phung, S.L.; Chai, D.; Bouzerdoum, A.; "Adaptive skin segmentation in color images," in: Acoustics, Speech, and Signal Processing, 2003. Proceedings. (ICASSP '03). 2003 IEEE International Conference. 6-10 April 2003, vol.3 pp 353-356.

[6] D. Petrescu and M. Gelgon "Face detection from complex Scenes in colour images," Eurasip/Proceedings/Eusipco2000.

[7] I.S. Hsieh, K.C. Fan and C. Lin, "A statistic approach to the detection of human faces in color nature scene," Pattern Recognition, 35(7)(2002) 1583-1596.

[8] Pentland, A.; Choudhury, T.; "Face recognition for smart environments," Feb 2000, pp 20-55.

[9] R.L. Hsu, M. Abdel-Mottaleb and A.K. Jain, "Face detection in color images," IEEE Trans. Pattern Analysis and Machine Intelligence, 24(5)(2002) 696-702.

[10] V. Vezhnevets, V. Sazonov and A. Andreeva, "A survey on pixelbased skin color detection techniques," in: Proc. Graphicon, Moscow, September 2003, pp. 85-92.

[11] S. L. Phung, A. Bouzerdoum and D. Chai, "Skin segmentation using color pixel classification: analysis and comparison," IEEE Transactions on Pattern Analysis and Machine Intelligence, 27(1)(2005) 148- 154

[12] B. Menser and M. Wien, "Segmentation and tracking of facial regions in color image sequences," Proc. SPIE VCIP, 2000, pp. 731-740.

[13] V. Vezhnevets, V. Sazonov, and A. Andreeva, "A Survey on Pixelbased Skin Color Detection Techniques,” Proc. Graphicon2003, Moscow, Russia, September 2003.

[14] J.-C. Terrillon, M. David, and S. Akamatsu, "Automatic Detection of Human Faces in Natural Scene Images by use of a Skin Color Model and of Invariant Moments," Proc. Int. Conf. AFGR'98, Nara, Japan, pp. 112-117, 1998.

[15] S.J. McKenna, S. Gong, and Y. Raja, "Modeling Facial Color and Identity with Gaussian Mixtures," Pattern Recognition, 31(12), pp. 1883-1892, 1998.

[16] R. Kjeldsen and J. Kender, "Finding Skin in Color Images," Proc. Int. Conf. AFGR'06, Killington, Vermont, pp. 312-317, 2006.

[17] M. J. Jones and J. M. Rehg, "Statistical color models with application to skin detection," Proc. CVPR'99, June 1999, pp. 274280.

[18] S. Gundimada, L. Tao, and V. Asari, "Face Detection Technique based on Intensity and Skin Color Distribution," ICIP2004, pp. 1413-1416, 2004.

[19] S.L. Phung, A. Bouzerdoum, and D. Chai, "A Novel Skin Color Model in YCbCr Color Space and its Application to Human Face Detection," ICIP2002, pp. 289-292, 2002.

[20] R.-L. Hsu, M. Abdel-Mottaleb, and A.K. Jain, "Face Detection in Color Images," IEEE Trans. PAMI, 24(5), pp.696-706, 2002.

[21] K. Sabottka and I. Pitas, "Segmentation and Tracking of Faces in Color Images,” AFGR'96, Killington, Vermont, pp. 236-241, 1996.

[22] P. Peer, J. Kovac, F. Solina, "Human Skin ColourClustering for Face Detection," EUROCON1993, Ljubljana, Slovenia, pp. 144148, September 2003.

[23] C. Garcia and G. Tziritas, "Face Detection Using Quantized Skin Color Regions Merging and Wavelet Packet Analysis," IEEE Trans. Multimedia, 1(3), pp. 264-277, 1999.

[24] Rafel C. Gonzalez, Richard E Woods, "Digital Image processing," Second edition Prentice Hall India.
[25] F. Fritsch, S. Lang, M. Kleinehagenbrock, G. A. Fink and Sagerer, "Improving Adaptive Skin Colour Segmentation by incorporating Results from face detection," Proceedings of IEEE International workshop on Robot and Human Interactive Communication (ROMAN), Berlin , Germany, September 2002.

[26] H.C. Vijaylakshmi, S. PatilKulakarni, "Face Localization and Detection Algorithm for Colour Images using Wavelet Approximations," International Conference ACVIT-09 16th - 19th December 2009, at Aurangabad. India.

[27] Pan Ng, Chi-Man Pun, "Skin color segmentation by Texture Feature Extraction and K-mean Clustering," in: Computational Intelligence, Communication Systems and Networks (CICSyN), 2011 Third International Conference, 26-28 July 2011, pp. 213218

[28] Mohammad Saber Iraji, Ali Yavari, "Skin Color Segmentation in Fuzzy YCBCR Color Space with the Mamdani Inference," in American Journal of Scientific Research, July (2011), pp.131137C EuroJournals Publishing, Inc. 2011.

[29] Bhatia, A.; Srivastava, S.; Agarwal, A.; "Face Detection Using Fuzzy Logic and Skin Color Segmentation in Images," in: Emerging Trends in Engineering and Technology (ICETET), 2010 3rd International Conference, 19-21 Nov. 2010, On page(s): 225 228.

[30] Lakshmi, H.C.V., PatilKulakarni, S.; "Segmentation Algorithm for Multiple Face Detection for Color Images with Skin Tone Regions," 9-10 Feb. 2010 , pp. 162 - 166,

[31] Ali Atharifard, and Sedigheh Ghofrani, "Robust Component-based Face Detection Using Color Feature," Proceedings of the World Congress on Engineering 2011 Vol II WCE 2011, July 6 - 8, 2011, London, U.K.

[32] Abbas Nasrabadi and Javad Haddadnia, "Skin Color Segmentation by Fuzzy Filter Applied to Face Detection," in International Journal of Computer and Electrical Engineering, Vol.2, No.6, December, 2010(1793-8163).

[33] H C Vijay Lakshmi and S. PatilKulakarni, "Segmentation Algorithm for Multiple Face Detection in Color Images with Skin Tone Regions using Color Spaces and Edge Detection Techniques," in International Journal of Computer Theory and Engineering, Vol. 2, No. 4, August, 2010(1793-8201). 